\title{
Light lon Beams for Energy Production in ADS
}

\author{
Mihaela Paraipan ${ }^{1,2, \star}$, Anton A. Baldin ${ }^{1,3}$, Elina G. Baldina ${ }^{1,3}$, and Serguey I. Tyutyunikov ${ }^{1}$ \\ 1 Joint Institute for Nuclear Research, Dubna, Russia \\ ${ }^{2}$ Institute of Space Science, Bucharest-Magurele, Romania \\ ${ }^{3}$ Institute for Advanced Studies "OMEGA", Dubna, Russia
}

\begin{abstract}
A comparative study of the energy efficiency of proton beams with an energy from $0.5 \mathrm{GeV}$ to $4 \mathrm{GeV}$ and light ion beams $\left({ }^{7} \mathrm{Li},{ }^{9} \mathrm{Be},{ }^{11} \mathrm{~B}\right.$, and $\left.{ }^{12} \mathrm{C}\right)$ with energies from $0.25 \mathrm{AGeV}$ to $1 \mathrm{AGeV}$ in natural and enriched quasi-infinite $\mathrm{U}$ target is presented. The numerical results on the particle transport and interaction are obtained using the code Geant4. The following target optimization issues are addressed: the beam window dimensions, and the possibility to use a core from low $\mathrm{Z}$ materials. The best solution for ADS from the point of view of the energy gain and miniaturization is obtained for ${ }^{7} \mathrm{Li}$ or ${ }^{9} \mathrm{Be}$ beam with an energy of $0.3-0.4 \mathrm{AGeV}$ and a target with Be core.
\end{abstract}

\section{Introduction}

The use of proton beams for energy production in accelerator-driven systems (ADS) was extensively investigated and the general opinion is that optimal for ADS is a proton beam with energy $\sim 1 \mathrm{GeV}$ [1]. The possibility to use heavy ion beams was less analyzed and the conclusions of authors are contradictory [2]-[3]. Our results presented in [4] show that one can get a higher efficiency by accelerating heavier ions. In the present work we analyse in more detail the use of light ion beams at energies below $1 \mathrm{AGeV}$.

\section{The energetic efficiency of the beam}

The beam efficiency is described by the energy gain factor $\mathrm{G}$ calculated as the ratio of the produced electrical power $P_{\text {prod }}$ to the power spent to accelerate the beam $P_{\text {spent }}$ :

$$
G=P_{\text {prod }} / P_{\text {spent }}
$$

The produced electrical power is:

$$
P_{\text {prod }}=\eta_{\mathrm{el}} \cdot E_{\mathrm{dep}} \cdot I_{\text {beam }},
$$

where $\eta_{\mathrm{el}}$ represents the conversion coefficient from thermal to electrical power, $E_{\mathrm{dep}}$ is the energy deposited in the target, and $I_{\text {beam }}$ is the beam intensity. The power spent for the beam acceleration $P_{\text {spent }}$ is analysed as the sum of two terms. The first term $P_{\text {beam }}$ represents the power transmitted to the

\footnotetext{
^e-mail: mihaela_paraipan@yahoo.com
} 
beam. The second term $P_{\text {acc }}$ contains the energy necessary to ensure the functioning of the accelerator (magnets, cooling system, etc.)

$$
P_{\text {spent }}=P_{\text {beam }}+P_{\text {acc }}=A \cdot E \cdot I_{\text {beam }}+P_{\text {acc }} .
$$

The term $P_{\text {acc }}$ depends on the type of the accelerator used. In a synchrotron this term scales as:

$$
P_{\mathrm{acc}}=\frac{A \cdot Z_{0} \cdot p}{A_{0} \cdot Z \cdot p_{0}} P_{\mathrm{acc}_{0}}
$$

In a linac $P_{\text {acc }}$ scales as:

$$
P_{\mathrm{acc}}=\frac{A \cdot Z_{0} \cdot E}{A_{0} \cdot Z \cdot E_{0}} P_{\mathrm{acc}_{0}}
$$

and in a cyclotron:

$$
P_{\mathrm{acc}}=\left(\frac{A \cdot Z_{0} \cdot p}{A_{0} \cdot Z \cdot p_{0}}\right)^{2} P_{\mathrm{acc}_{0}}
$$

In formulas (3)-(6) $A, Z, p$ and $E$ are the mass number, the atomic number, the momentum per nucleon and the energy per nucleon of the analysed particle. The symbols with the subscript 0 represent the corresponding values for the reference particle. These formulas allow to calculate the power spent for any beam if we know the accelerator efficiency for a reference beam.

\section{The energy produced in the target}

The energy released in the target was calculated with the code Geant 4 . The hadronic inelastic interaction of protons, neutrons and pions was modelled with the Bertini cascade model. For neutrons with energies below $20 \mathrm{MeV}$ high precision neutron models based on a detailed implementation of the data from ENDF (Evaluated Nuclear Data File) were used. The inelastic interaction of ions was modelled with the binary cascade model.

Cylindrical targets with dimensions large enough in order to ensure quasi-infinite conditions (the saturation of the energy deposited in both radial and longitudinal directions) were used. In the case of ${ }^{\text {nat }} U$ target the radius is $60 \mathrm{~cm}$ and the length $160 \mathrm{~cm}$. The ${ }^{\text {nat }} U$ target has a low critical coefficient (0.48) and needs very high beam intensities for efficient power production. An enriched target with critical coefficient 0.96 ensures a safe exploitation and needs beam intensities 50 times lower. In such target $\left(5.7 \%{ }^{235} \mathrm{U}\right)$ the quasi-infinite conditions are reached at higher dimensions. In this case a cylinder with radius $100 \mathrm{~cm}$ and length $200 \mathrm{~cm}$ was used. The influence of the the beam window length on the energy released was investigated. The presence of beam window increases the energy deposited by including the effect from backscattered particles. The simulation shows that the optimum length of the beam window is $\sim 40 \mathrm{~cm}$.

For ion beams at low energies the possibility to use low $\mathrm{Z}$ materials in the central part of the target was considered. Two phenomena with opposite effects concur in this case. On one side in a low $\mathrm{Z}$ material the energy loss through ionization decreases and the probability of inelastic interaction at higher energy per nucleon increases, generating higher multiplicity and a harder spectrum of the secondaries. On the other side a low mass number of the target diminishes the cross section. The overall result depends on which of these effects dominates. The simulation predicts that a core from very low $\mathrm{Z}$ materials $(\mathrm{Li}, \mathrm{Be}, \mathrm{C})$ increases the energy released for light ions at low energies with a factor of 1.3-2. The maximum effect is obtained with a core of Be. 


\section{Results and discussion}

The first set of simulations was performed in a ${ }^{\text {nat }} U$ target irradiated with protons and ions with masses until ${ }^{40} \mathrm{Ca}$ and energies from $0.3 \mathrm{AGeV}$ to $10 \mathrm{AGeV}$. The dependence of the energy released on the projectile mass and energy per nucleon is presented in figure 1a. The relative beam efficiency for different types of accelerators is also given (figure 1b-d). As reference beam we use protons with energy $1 \mathrm{GeV}$ for which a value of 0.1 for the accelerator efficiency was assumed. When accelerated in a synchrotron or linac ion beams starting with ${ }^{7} \mathrm{Li}$ demonstrate higher energetic efficiency than protons. The maximum efficiency for ions with higher masses is reached at higher energies. That does not represent a problem when the beam is accelerated in a synchrotron, but such energies are difficult to reach in a linear accelerator, needing accelerator lengths of a few $\mathrm{km}$. Unfortunately, the beam intensities achieved in a synchrotron are by orders of magnitude lower than those necessary for ADS. Linear accelerators look more promising for obtaining the required beam intensity.
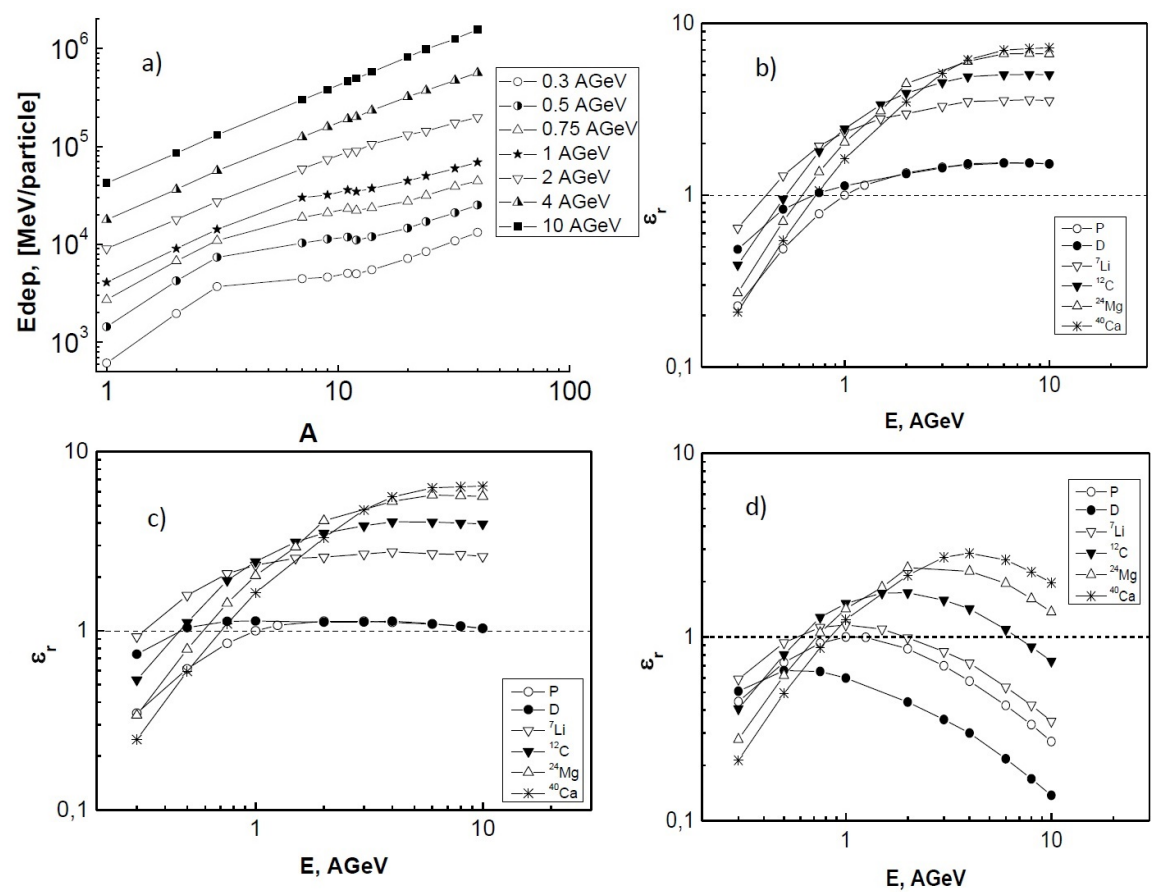

Figure 1. The energy released as function on the projectile type and energy (a), the relative efficiency for beams accelerated in a synchrotron (b), linear accelerator (c), and cyclotron (d)

For this reason in the second set of simulations we realized in enriched $\mathrm{U}$ target irradiated with light ions from ${ }^{7} \mathrm{Li}$ to ${ }^{12} \mathrm{C}$ at low energies $(0.3 \mathrm{AGeV}-1 \mathrm{AGeV})$, accelerated in a linac. We use the data from the European Spallation Source (ESS) project for the reference particle (protons with final energy $2.5 \mathrm{GeV}$, beam intensity $I_{0}$ of $1.25 \cdot 10^{16} \mathrm{p} / \mathrm{s}$, and a value of 0.18 for the accelerator efficiency). The total energy deposited per projectile, the energy gain and the net electrical power obtained with different beams at the same intensity $I_{0}$ are presented in table 1 for a target entirely from enriched $\mathrm{U}$ and with core of Be.

In target with $\mathrm{Be}$ core beams of ${ }^{7} \mathrm{Li}$ or ${ }^{9} \mathrm{Be}$ are equivalent from the point of view of the energy produced with a beam of $1.5 \mathrm{GeV}$ protons (at maximum proton beam efficiency) but with higher energy gain and needing accelerator with lower dimensions. 
Table 1. The energy released $E_{\mathrm{dep}}$, the gain factor $G$ and the net electrical power $P_{\mathrm{el}}$ produced in enriched $\mathrm{U}$ target irradiated with protons and light ions

\begin{tabular}{llllllll}
\hline & \multicolumn{3}{c}{ Fuel } & \multicolumn{3}{c}{ Core Be } \\
\hline$P$ & $E_{\text {beam }}$ & $E_{\text {dep }}$ & $\mathrm{G}$ & $P_{\text {el }}$ & $E_{\text {dep }}$ & $G$ & $P_{\text {el }}$ \\
& AGeV & MeV & & MW & MeV & & MW \\
proton & 1 & $1.09 \mathrm{e} 5$ & 7.3 & 75.6 & $1.25 \mathrm{e} 5$ & 8.64 & 88.1 \\
& 1.5 & $1.85 \mathrm{e} 5$ & 8.23 & 130.2 & $2.18 \mathrm{e} 5$ & 9.68 & 156.3 \\
& 2 & $2.49 \mathrm{e} 5$ & 8.32 & 175.6 & $2.94 \mathrm{e} 5$ & 9.8 & 211.2 \\
${ }^{7} \mathrm{Li}$ & 0.3 & $8.49 \mathrm{e} 4$ & 6.06 & 56.7 & $1.79 \mathrm{e} 5$ & 12.8 & 132.1 \\
& 0.4 & $1.54 \mathrm{e} 5$ & 8.19 & 107.3 & $3.21 \mathrm{e} 5$ & 17.2 & 242.1 \\
& 0.5 & $2.39 \mathrm{e} 5$ & 10.3 & 172.9 & $4.65 \mathrm{e} 5$ & 19.9 & 353.1 \\
& 0.75 & $4.99 \mathrm{e} 5$ & 14.3 & 371.4 & $7.7 \mathrm{e} 5$ & 22 & 588.1 \\
& 1 & $7.19 \mathrm{e} 5$ & 15.4 & 537.7 & $1.07 \mathrm{e} 6$ & 22.8 & 814.7 \\
${ }^{9} \mathrm{Be}$ & 0.4 & $1.5 \mathrm{e} 5$ & 7.39 & 120 & $3.34 \mathrm{e} 5$ & 16.5 & 267 \\
& 0.5 & $2.5 \mathrm{e} 5$ & 9.86 & 200 & $5.3 \mathrm{e} 5$ & 20.9 & 424 \\
${ }^{11} \mathrm{~B}$ & 0.5 & $2.26 \mathrm{e} 5$ & 8.17 & 158 & $5.21 \mathrm{e} 5$ & 18.9 & 417 \\
& 0.75 & $5.22 \mathrm{e} 5$ & 12.7 & 385.1 & $1.08 \mathrm{e} 6$ & 26.2 & 863 \\
${ }^{12} \mathrm{C}$ & 0.3 & $5.5 \mathrm{e} 4$ & 3.3 & 30.8 & $1.22 \mathrm{e} 5$ & 7.4 & 84.7 \\
& 0.4 & $1.08 \mathrm{e} 5$ & 4.9 & 68.6 & $2.62 \mathrm{e} 5$ & 11.9 & 191.9 \\
& 0.5 & $1.93 \mathrm{e} 5$ & 7 & 32.5 & $4.68 \mathrm{e} 5$ & 17 & 352.1 \\
& 0.75 & $4.8 \mathrm{e} 5$ & 11.6 & 351.3 & $1.03 \mathrm{e} 6$ & 25 & 793.4 \\
& 1 & $8.07 \mathrm{e} 5$ & 14.7 & 601.7 & $1.5 \mathrm{e} 6$ & 27.3 & 1155.2
\end{tabular}

\section{Conclusions}

The optimal energy of proton beam depends on the accelerator type and is $\sim 3 \mathrm{GeV}$ in synchrotron, $1.5 \mathrm{GeV}$ in linac, and $1 \mathrm{GeV}$ in cyclotron. The optimal energy for ion beams depends on the type of the ion (1.5-2 AGeV for ${ }^{7} \mathrm{Li}, 2 \mathrm{AGeV}$ for ${ }^{12} \mathrm{C}, 4 \mathrm{AGeV}$ for ${ }^{40} \mathrm{Ca}$ ) and the efficiency is significantly higher (more than 2 times) than for protons.

Light ions ${ }^{7} \mathrm{Li}$ and ${ }^{9} \mathrm{Be}$ with energy $0.3-0.35 \mathrm{AGeV}$ realize the same energy release as a beam of proton $1.5 \mathrm{GeV}$. This allows one to obtain the same electrical power with lower energy consumption and an accelerator with $\sim 2$ times lower dimensions. The acceleration of ${ }^{11} \mathrm{~B}$, and ${ }^{12} \mathrm{C}$ at $0.7-0.75$ $\mathrm{AGeV}$ needs an accelerator with the same dimensions as for proton beam $1.5 \mathrm{GeV}$ but produces a net electrical power about 5 times higher.

The best solution from the point of view of the energy gain and miniaturization is the ${ }^{7} \mathrm{Li}$ beam with an energy of $0.3 \mathrm{AGeV}$ and a target with core of Be.

\section{References}

[1] C. Rubbia et al., preprint CERN/AT/95-44 (ET) (1995)

[2] D. G. Koshkarev, N. M. Sobolevski, and A. V. Barhudarian, Atomic Energy 105 (3), 137-139 (2008)

[3] S. R. Hashemi-Nezhad, W. Westmeier, M. Zamani-Valasiadou, B. Thomauske, and R. Brandt, Ann. Nucl. Energy 38, 1144-1155 (2011)

[4] A. A. Baldin, A. I. Berlev, M. Paraipan, and S. I. Tyutyunikov, Phys. Part. Nucl. Let. 14 (1), $113-119$ (2017) 